\title{
Expression profile analysis of head and neck squamous cell carcinomas using data from The Cancer Genome Atlas
}

\author{
LI YAN $^{1}$, CHENG ZHAN $^{2}$, JIHONG WU ${ }^{3}$ and SHENGZI WANG ${ }^{1}$ \\ ${ }^{1}$ Department of Radiation Oncology, Eye \& ENT Hospital, Fudan University, Shanghai 200031; \\ ${ }^{2}$ Department of Thoracic Surgery, Zhongshan Hospital, Fudan University, Shanghai 200032; \\ ${ }^{3}$ Research Center, Eye \& ENT Hospital, Fudan University, Shanghai 200031, P.R. China
}

Received May 4, 2015; Accepted March 7, 2016

DOI: $10.3892 / \mathrm{mmr} .2016 .5054$

\begin{abstract}
Head and neck squamous cell carcinoma (HNSCC) is the major histological type of head and neck cancer and no curative treatments are currently available. Using advanced sequencing technologies, The Cancer Genome Atlas (TCGA) has produced large-scale sequencing data, which provide unprecedented opportunities to reveal molecular mechanisms of cancer. The present study analyzed the mRNA and micro (mi)RNA expression data of HNSCC and normal control tissues released by the TCGA database using a bioinformatics approach to explore underlying molecular mechanisms. The mRNA and miRNA expression data were downloaded from the TCGA database and differentially expressed genes (DEGs) and miRNAs (DEMs) between HNSCC and normal head and neck tissues were identified using TwoClassDif. Subsequently, the gene functions and pathways which are significantly altered in HNSCC were identified using Gene Ontology (GO) and Kyoto Encyclopedia of Genes and Genomes (KEGG) pathway enrichment analysis. Regulatory networks among DEGs and DEMs were then constructed, and transcription factors (TFs) potentially regulating the DEGs and DEMs were determined and a TF - miRNA - gene network was established. A total of 2,594 significant DEGs (1,087 upregulated and 1,507 downregulated), and 25 DEMs (8 upregulated and 17 downregulated) were identified in HNSCC compared with normal control samples. These DEGs were significantly enriched in GOs and KEGG pathways such as mitosis, cell cycle, Wnt, JAK/STAT and TLR signaling pathway. CPBP, NF-AT1 and miR-1 were situated
\end{abstract}

Correspondence to: Professor Shengzi Wang, Department of Radiation Oncology, Eye \& ENT Hospital, Fudan University, 83 Fenyang Road, Xuhui, Shanghai 200031, P.R. China

E-mail: shengziwang@fudan.edu.cn

Key words: head and neck squamous cell carcinomas, differentially expressed genes, differentially expressed microRNAs, gene ontology analysis, Kyoto Encyclopedia of Genes and Genomes analysis, transcription factor - microRNA - gene network, the cancer genome atlas in the central hub of the TF - miRNA - gene network, underlining their central roles in regulatory processes specific for HNSCC. The present study enhanced the current understanding of the molecular mechanisms underlying HNSCC and may offer novel strategies for its prevention, diagnosis and treatment.

\section{Introduction}

Head and neck squamous cell carcinoma (HNSCC), the major histological type of head and neck cancer (90-95\%), is the sixth most common cancer type worldwide, with an incidence of $<600,000$ cases per year $(1,2)$. More than $60 \%$ of HNSCC patients are already in the advanced stage at the time-point of first diagnosis (3). Despite the advances in the treatments for HNSCC, including surgical methods, chemoradiotherapy and the introduction of targeted therapies, the overall survival has not significantly improved in the last decades, with a five-year survival rate of only $40-50 \%$ (10-30\% for patients with stage IVa and IVb tumors) $(4,5)$. Therefore, it is urgently required to thoroughly explore the molecular characteristics of HNSCC to develop novel molecular-based targeted strategies for its treatment.

Numerous recent studies have investigated the biological characteristics of HNSCC. Certain genes, micro (mi)RNAs, transcription factors (TFs) and signaling pathways, including XPA, miR-504, EGR3 and the Notch signaling pathway, respectively, have important roles in the genesis and development of HNSCC, and are correlated to the prognosis of affected patients (6-8). However, to date, a comprehensive and systemic analysis of expression profiles in HNSCC has been lacking. The present study used the high-throughput mRNA and miRNA expression data from hundreds of HNSCC samples released by The Cancer Genome Atlas (TCGA) database to identify differentially expressed genes (DEGs) and miRNAs (DEMs) between human HNSCC and normal head and neck tissues. Significant gene functions and signaling pathways in which those DEGs are enriched were then determined. Finally, the regulatory network among TFs, DEMs and DEGs was mapped. The present study contributed to the current understanding of the molecular basis of HNSCC and may aid in the development of novel means of prevention, diagnosis and treatment. 


\section{Materials and methods}

Data sources. Level 3 RNA-sequencing data from 43 normal samples and 498 HNSCC samples, and level 3 miRNA-sequencing data from 45 normal samples and 513 HNSCC samples released by TCGA prior to July 1st, 2014 were all obtained from the TCGA data portal (https://tcga-data. nci.nih.gov/tcga/). As published previously, the mature miRNAs and star miRNAs (3' arms of pre-miRNA) from each pre-miRNA in the miRNA sequencing data were sorted and calculated according to their MIMAT serial number based on miRbase V20.0 (http://www.mirbase.org), whereas stem-loop, precursor or unannotated transcript data were not included in the present analysis $(9,10)$. Reads per kilobase of exon model per million mapped reads (RPKM) and reads per million miRNA mapped (RPM) values were used to represent mRNA and miRNA expression levels, respectively (11). All data were presented as the mean \pm standard deviation.

Identification of DEGs and DEMs. TwoClassDif was used to identify DEGs and DEMs between normal samples and HNSC samples as previously reported $(12,13)$. Briefly, Fold-change (Tumor/Normal) was firstly used to filter DEGs and DEMs. Only genes with a Fold-change (Tumor/Normal) of $>2$ or $<0.5$, and miRNAs with a Fold-change (Tumor/Normal) of $>2.5$ or $<0.4$ progressed to next stages. Subsequently, the DEGs and DEMs were further confirmed with the t-test and random variance model (RVM)-modified t-test to reduce statistical errors using SPSS for windows, version 20 (International Business Machines, Armonk, NY, USA). In the present study, $\mathrm{P}<0.05$ corrected by the false discovery rate (FDR) was considered to indicate a statistically significant difference.

Gene ontology (GO) analysis. GO analysis was performed according to the GO database (http://geneontology.org/) as previously described $(14,15)$. In brief, the $\chi^{2}$ test and Fisher's exact test were used to test the significance level of each function, and the FDR was calculated to correct statistical errors derived from multiple tests. The significance threshold was set at $\mathrm{P}<0.01$ and FDR $<0.05$. The results were then classified in a GO-map to further integrate the functional links between these significant GO functions using Cytoscape v3.2.0 (http://cytoscape.org/).

Kyoto Encyclopedia of Genes and Genomes (KEGG) analysis. KEGG analysis was performed as described previously using the KEGG database (http://www.genome. $\mathrm{jp} / \mathrm{kegg} /$ ) (16-18). The $\chi^{2}$ test and Fisher's exact test were also performed to screen the significant pathways, and the P-value, FDR and enrichment value were calculated as previously reported (17). Pathways with $\mathrm{P}<0.01$ and $\mathrm{FDR}<0.05$ were considered as significant. Then, based on the pathway associations in the KEGG database, the path network was established to integrate the interactions among these significant pathways using Cytoscape v3.2.0.

TF - miRNA - gene network analysis. First, the target genes of the DEMs were predicted using Targetscan (http://www.targetscan.org/) and Miranda (http://www. microrna.org/) $(19,20)$. The intersections of predictions from the two databases were matched to these DEGs to identify their regulatory targets.

To predict the TFs that regulate these DEGs and DEMs, the DNA sequences of these genes and pre-miRNA near the transcription start site area were acquired, including 1,000-bp upstream and 200-bp downstream regions. Then, the Match ${ }^{\mathrm{TM}}$ algorithm in the TRANSFAC database (http://www.gene-regulation.com/index2) was used to search for TF binding sites in these regions. Two score values generated in this algorithm, the core similarity score (CSS) and the matrix similarity score (MSS), were then applied to evaluate the forecast results (21-23). Based on these results, a TF - miRNA - gene network was finally constructed using Cytoscape v3.2.0, to summarize and illustrate the regulatory interactions among TFs, DEMs and DEGs.

\section{Results}

Identification of DEGs and DEMs. The present study identified 2,594 significant DEGs (1,087 upregulated and 1,507 downregulated), and 25 significant DEMs (8 upregulated and 17 downregulated) in HNSCC compared with normal control samples. The DEGs and DEMs with the greatest fold-change are shown in Fig. 1.

GO analysis results. GO analysis was performed to preliminarily summarize the biological functions of the DEGs (Fig. 2).

The upregulated genes were mainly enriched in GO terms including mitosis (GO:0007067), cell proliferation (GO:0008283), cell division (GO 0051301) and the cell cycle (GO:0007049), which are features accountable for the overproliferation of HNSCC cells. They were also enriched in cell migration (GO:0016477), extracellular matrix organization (GO:0030198), cell-cell signaling (GO:0007267), angiogenesis (GO:0001525) and vascular endothelial growth factor receptor signaling pathway (GO:0048010), indicating the metastatic and angiogenic capacities of HNSCC.

Downregulated genes were mainly involved in the G-protein coupled receptor signaling pathway (GO:0007186), epidermal growth factor receptor signaling pathway (GO:0007173), transmembrane transport (GO:0055085) and glucose metabolic processes (GO:0006006), which indicated the potential negative effects of these GO terms in the genesis and development of HNSCC.

Furthermore upregulated, as well as downregulated, genes were significantly enriched in processes including cell differentiation (GO:0030154), cell adhesion (GO:0007155), immune response (GO:0006955) and ion transport (GO:0006811), suggesting that intricate changes were likely to occur in these GOs during the genesis of HNSCC.

Aberrant pathways in HNSCC tissues. As displayed in Fig. 3, the upregulated genes in HNSCC were significantly enriched in the cell cycle (Path ID: 4110), Wnt signaling pathway (Path ID: 04310), p53 signaling pathway (Path ID: 04115), Jak/STAT signaling pathway (Path ID: 04630), TGF- $\beta$ signaling pathway (Path ID: 04350), Toll-like receptor signaling pathway (Path ID: 04620) and extracellular matrix - receptor interaction (Path ID: 04512). 

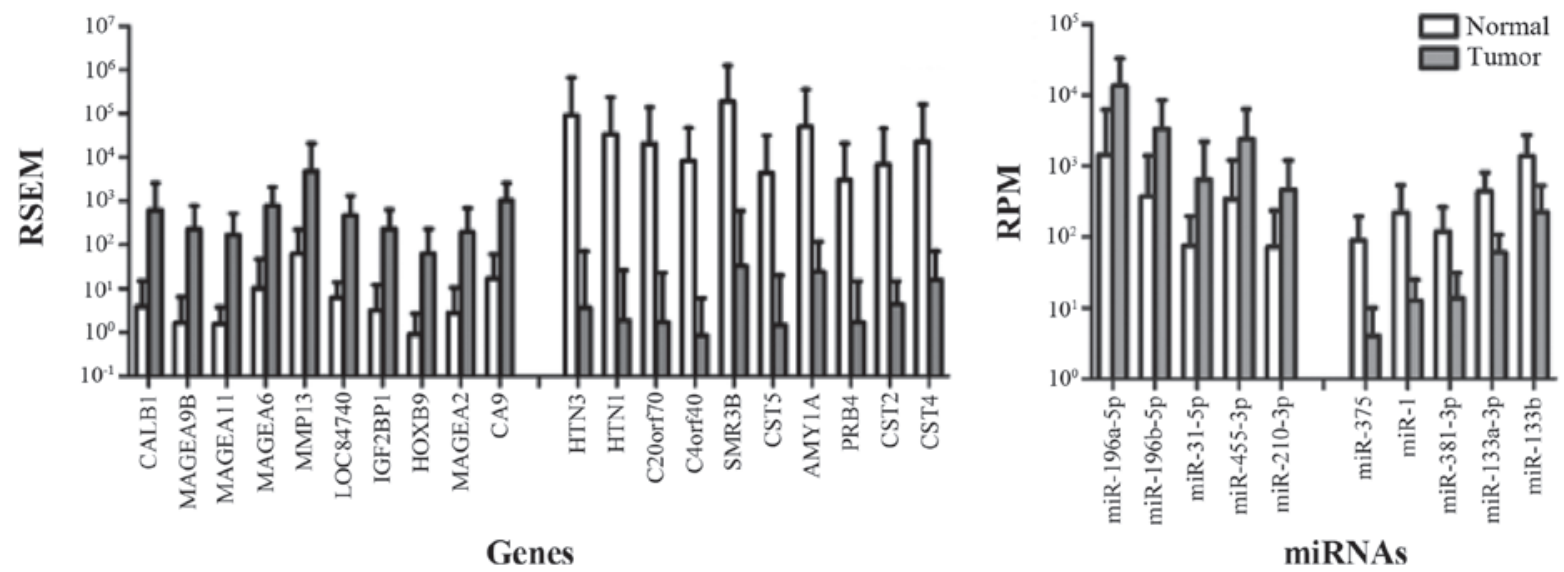

Figure 1. Top-10 differentially expressed genes and top-5 differentially expressed miRNAs in head and neck squamous cell carcinomas ordered by fold-change. miRNA/miR, microRNA; RSEM, RNA-Seq by expectation maximization; RPM, Reads per million miRNAs mapped.

The downregulated genes mostly participated in the calcium signaling pathway (Path ID: 04020), ErbB signaling pathway (Path ID: 04012), GnRH signaling pathway (Path ID: 04912), pyruvate metabolism (Path ID: 00620) and further small molecular metabolism pathways, while the upregulated as well as downregulated genes were enriched in pathways including the MAPK signaling pathway (Path ID: 4010) and cell adhesion molecules (Path ID: 04514).

TF - miRNA - gene network. As shown in Fig. 4, CPBP (also called KLF6) was located in the central hub of the TF - miRNA - gene network and regulated the largest number of DEMs $(n=22)$ and genes $(n=97)$ in HNSCC. This was followed by NF-AT1 (also termed NFATC2), GKLF (also known as KLF4), ZNF333 and Churchill. miR-1, miR-101-3p, miR-486-5p, miR-133a-3p and miR-195-5p were the top-5 DEMs that regulated the highest number of DEGs. These molecular regulators were indicated to have key roles in HNSCC.

\section{Discussion}

The present study identified 2,594 DEGs and 25 DEMs in HNSCC based on gene and miRNA expression profiles of HNSCC tissues compared with normal tissues. A large variety of DEMs, including CALB1 and MAGEA9B, DEMs, including miR-196a-5p and miR-196b-5p, in HNSCC were identified. Overexpression of CALB1 has been previously reported to be inversely correlated with apoptosis to be correlated with poor prognosis in other cancer types $(24,25)$. MAGEA9B and three other genes belonging to the melanoma antigen family A (MAGEA) were among the ten most strongly upregulated genes of the present study. This gene family is highly expressed in early embryos and is associated with reduced overall survival in numerous types of cancer (26-28). However, the roles of the CALB1 and MAGEA gene families have been rarely studied in HNSCC and require to be further investigated. As the most strongly upregulated miRNAs in the present study, miR-196a-5p and miR-196b-5p have been frequently reported to be overexpressed in the blood of HNSCC patients and in HNSCC tissues and to be associated with the prognosis and radio-response, indicating their potential as promising diagnostic and prognostic biomarkers as well as therapeutic targets (29-33). Therefore, the potential of these DEGs and DEMs as biomarkers and therapeutic targets deserve further investigation with regard to their application in the early diagnosis, pathological identification, treatment and monitoring of HNSCC.

To explore the main functional enrichment and signaling pathways of these DEGs at the cellular level, GO and KEGG pathway analyses were performed, respectively. GO functions and pathways including mitosis and cell cycle as well as Wnt, JAK/STAT and Toll-like receptor signaling pathways were markedly altered in HNSCC compared with normal controls. The mutation, abnormal expression and modification of these GOs and pathways have been frequently reported in HNSCC and other cancer types (34-38). In tumor cells, the normal energy metabolism of aerobic respiration is replaced by glycolysis, which is harnessed for accumulating intermediates of macromolecule biosynthesis, known as the 'Warburg effect' (39-41). Along with this, the present study revealed that the downregulated DEGs were significantly enriched in gene functions and pathways associated with the metabolism of glucose, pyruvate and numerous other small molecules. These results suggested that these significant GOs and pathways are critical drivers in the carcinogenesis and development of HNSCC, and so are the DEGs that participate in these GOs and pathways. As shown in the interaction networks, considerable cross-links exist among these gene functions and pathways, and therapeutic targeting of one of them may modulate others, indicating that several parts of the network may be affected by targeting one component for the treatment of HNSCC. The hub genes involved in the significant GO and pathway networks might be applied as novel targets in HNSCC therapeutic strategies.

There are two major ways in which cells generally regulate gene expression; one is to regulate the transcription from DNA to RNA via elements, such as TFs, while the other is to regulate the stability of RNA via factors, such as miRNAs (42). Using the mRNA and corresponding miRNA expression data released by TCGA, the present study constructed the TF - miRNA - gene network in HNSCC. In this network, 


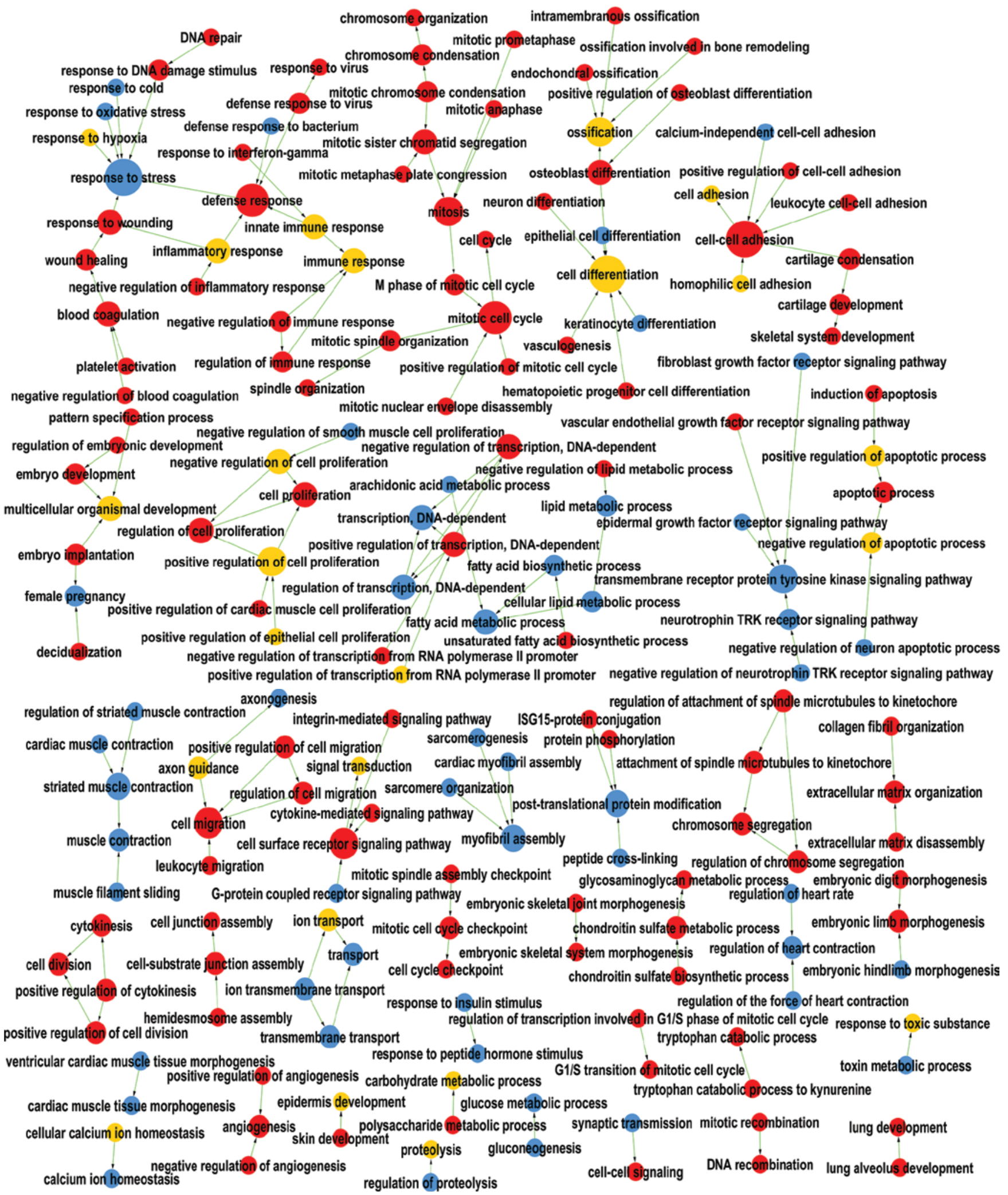

Figure 2. Gene ontology map for head and neck squamous cell carcinomas. Circles represent genetic functions of differentially expressed genes, with their size being proportional to the importance of their function. Red represents functions encoded by upregulated genes, blue represents functions encoded by downregulated genes and yellow represents functions encoded by up- as well as downregulated genes. Straight lines represent links between genetic functions.

CPBP, NF-AT1 and miR-1 were situated in the central hub, indicating their marked importance in the regulatory net of HNSCC. CPBP is a member of the Kruppel-like family of TFs, some of which are implicated in carcinogenesis, acting in processes ranging from cell proliferation and apoptosis to differentiation, migration and pluripotency (43). NF-AT1 has a central role in gene transcription during the immune response and is associated with several tumor types, including glio- 


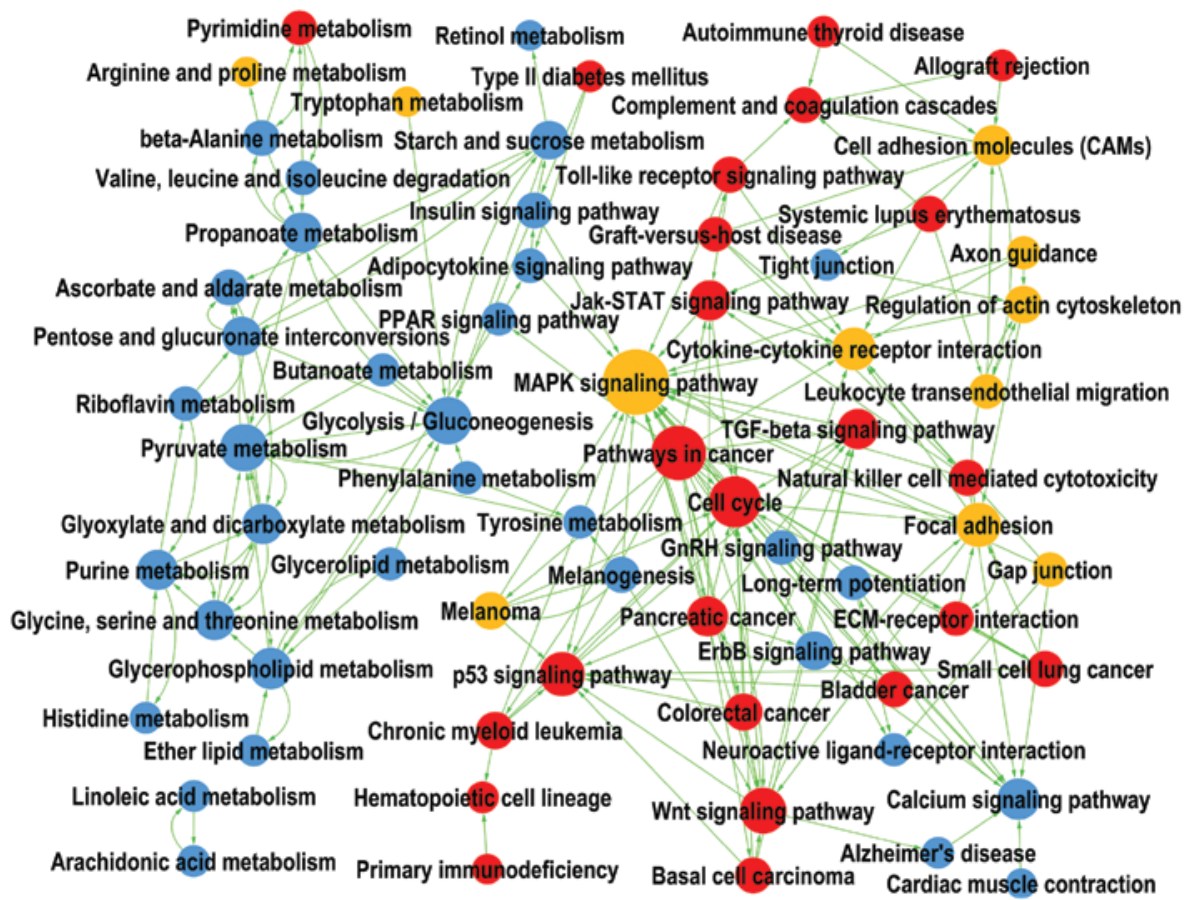

Figure 3. Kyoto Encyclopedia of Genes and Genomes path network in head and neck squamous cell carcinomas. Circles represent pathways, and their size is relative to the importance of the respective pathway. Red represents pathways of upregulated genes, blue represents pathways of downregulated genes and yellow represents pathways of up- as well as downregulated genes. Straight lines represent interactions between pathways.

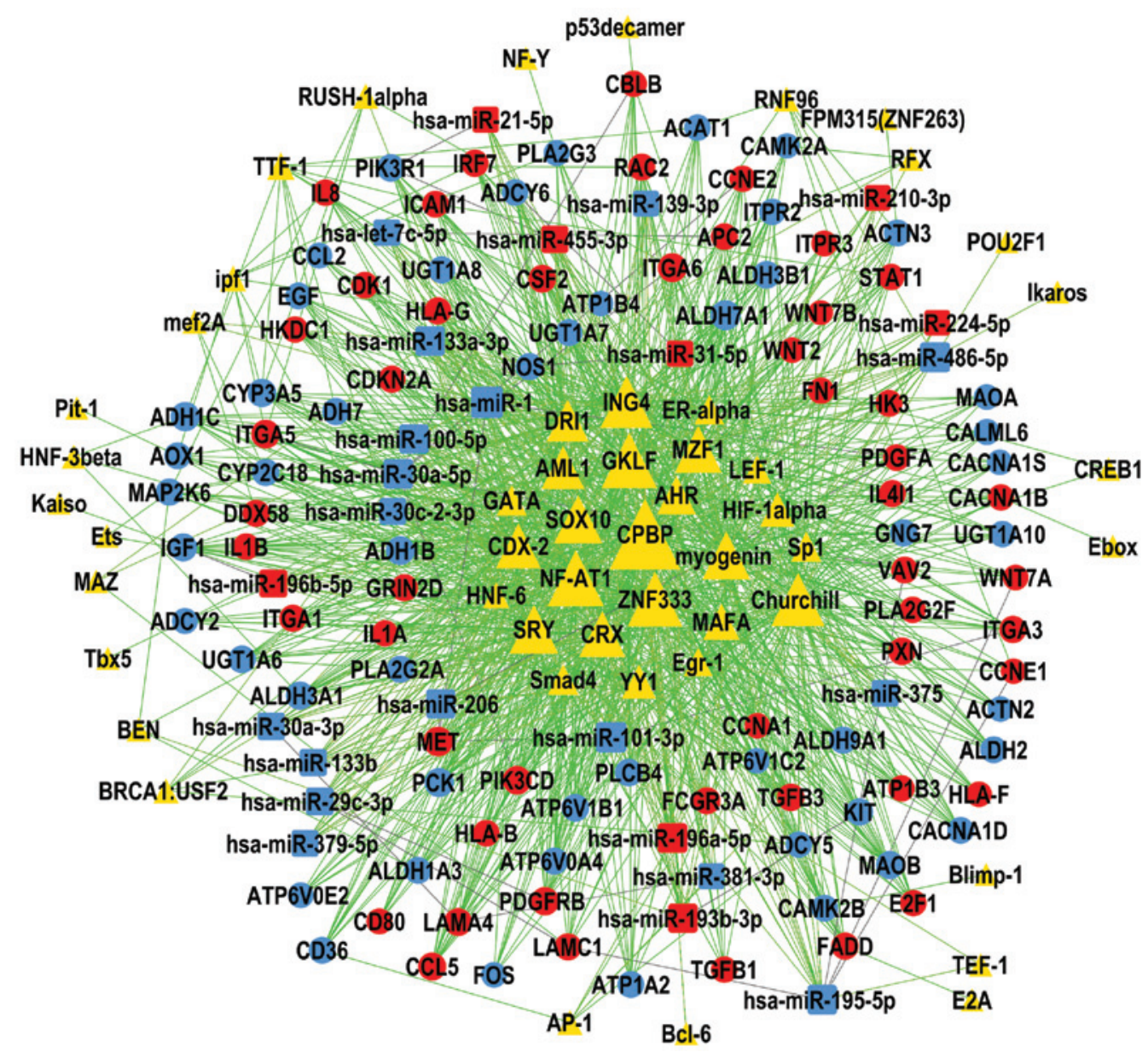

Figure 4. TF - miRNA - gene regulation network in head and neck squamous cell carcinomas. Triangles represent TFs, circles represent differentially expressed genes and rounded rectangles represent differentially expressed miRNAs, with their area being relative to the significance. Red represents upregulation, blue represents downregulation and yellow represents up- as well as downregulation. Straight lines represent regulatory interactions. TF, transcription factor; miR, microRNA. 
blastoma and human melanoma $(44,45)$. However, the roles of these two TFs in have remained elusive in HNSCC. As the second most downregulated DEM, miR-1 has been reported to be a tumor suppressor miRNA targeting transgelin 2, purine nucleoside phosphorylase, fibronectin 1 and prothymosin alpha, and to accelerate apoptosis and inhibit proliferation in HNSCC (46-49). Further investigation on these TFs and miRNAs will enhance the current understanding of the molecular mechanisms of HNSCC and help to identify potential therapeutic targets for the treatment of HNSCC.

Based on the mRNA and corresponding miRNA expression data for hundreds of HNSCC samples, the present study identified the DEGs and DEMs, and then investigated the GOs and pathways in which the DEGs were significantly enriched. The regulatory links among the DEMs and DEGs were determined and potential TFs, which regulate these DEMs and DEGs were predicted to finally construct the TF - miRNA - gene network. Additionally, the hub genes, TFs and miRNAs may potentially be targeted by novel therapeutic strategies in the future. To the best of our knowledge, the present study was the first systematic bioinformatics analysis in HNSCC, and for the first time, significantly altered GOs and pathways, as well as DEMs and TFs were identified in HNSCC. The results of the present study enhanced the current understanding of the molecular mechanisms underlying HNSCC, and may provide a source for developing novel strategies for its prevention, diagnosis and treatment.

\section{Acknowledgements}

The authors would like to thank the language editing service, ICE-editing Co. for editing this manuscript. The present study was supported by The Science and Technology Commission Foundation of Shanghai (grant no. 12JC1402102).

\section{References}

1. Bose P, Brockton NT and Dort JC: Head and neck cancer: From anatomy to biology. Int J Cancer 133: 2013-2023, 2013.

2. Parkin DM, Bray F, Ferlay J and Pisani P: Global cancer statistics, 2002. CA Cancer J Clin 55: 74-108, 2005.

3. Seiwert TY, Salama JK and Vokes EE: The chemoradiation paradigm in head and neck cancer. Nat Clin Pract Oncol 4: 156-171, 2007.

4. Hsieh JC, Lin HC, Huang CY, Hsu HL, Wu TM, Lee CL, Chen MC, Wang HM and Tseng CP: Prognostic value of circulating tumor cells with podoplanin expression in patients with locally advanced or metastatic head and neck squamous cell carcinoma. Head Neck 37: 1448-1455, 2015.

5. Vokes EE, Weichselbaum RR, Lippman SM and Hong WK: Head and neck cancer. N Engl J Med 328: 184-194, 1993.

6. Mazumder TH, Nath S, Nath N and Kumar M: Head and neck squamous cell carcinoma: Prognosis using molecular approach Cent Eur J Biol 9: 593-613, 2014

7. Kikkawa N, Kinoshita T, Nohata N, Hanazawa T, Yamamoto N, Fukumoto I, Chiyomaru T, Enokida H, Nakagawa M, Okamoto Y and Seki N: microRNA-504 inhibits cancer cell proliferation via targeting CDK6 in hypopharyngeal squamous cell carcinoma. Int J Oncol 44: 2085-2092, 2014.

8. Brooks YS, Ostano P, Jo SH, Dai J, Getsios S, Dziunycz P, Hofbauer GF, Cerveny K, Chiorino G, Lefort K and Dotto GP: Multifactorial ER $\beta$ and NOTCH1 control of squamous differentiation and cancer. J Clin Invest 124: 2260-2276, 2014.

9. Zhan C, Yan L, Wang L, Jiang W, Zhang Y, Xi J, Chen L, Jin Y, Qiao Y, Shi Y and Wang Q: Identification of reference miRNAs in human tumors by TCGA miRNA-seq data. Biochem Biophys Res Commun 453: 375-378, 2014.
10. Kozomara A and Griffiths-Jones S: miRBase: Annotating high confidence microRNAs using deep sequencing data. Nucleic Acids Res 42 (Database issue): D68-D73, 2014.

11. Mortazavi A, Williams BA, McCue K, Schaeffer L and Wold B: Mapping and quantifying mammalian transcriptomes by RNA-Seq. Nat Methods 5: 621-628, 2008.

12. Clarke R, Ressom HW, Wang A, Xuan J, Liu MC, Gehan EA and Wang Y: The properties of high-dimensional data spaces: Implications for exploring gene and protein expression data. Nat Rev Cancer 8: 37-49, 2008.

13. Wright GW and Simon RM: A random variance model for detection of differential gene expression in small microarray experiments. Bioinformatics 19: 2448-2455, 2003.

14. Ashburner M, Ball CA, Blake JA, Botstein D, Butler H, Cherry JM, Davis AP, Dolinski K, Dwight SS, Eppig JT, et al: Gene ontology: Tool for the unification of biology. The Gene Ontology Consortium. Nat Genet 25: 25-29, 2000.

15. Harwood CR and Moszer I: From gene regulation to gene function: Regulatory networks in Bacillus subtilis. Comp Funct Genomics 3: 37-41, 2002.

16. Yi M, Horton JD, Cohen JC, Hobbs HH and Stephens RM: WholePathwayScope: A comprehensive pathway-based analysis tool for high-throughput data. BMC Bioinformatics 7: 30, 2006.

17. Kanehisa M, Goto S, Kawashima S, Okuno Y and Hattori M: The KEGG resource for deciphering the genome. Nucleic Acids Res 32 (Database issue): D277-D280, 2004.

18. Draghici S, Khatri P, Tarca AL, Amin K, Done A, Voichita C, Georgescu C and Romero R: A systems biology approach for pathway level analysis. Genome Res 17: 1537-1545, 2007.

19. Garcia DM, Baek D, Shin C, Bell GW, Grimson A and Bartel DP: Weak seed-pairing stability and high target-site abundance decrease the proficiency of lsy- 6 and other microRNAs. Nat Struct Mol Biol 18: 1139-1146, 2011.

20. Betel D, Koppal A, Agius P, Sander C and Leslie C: Comprehensive modeling of microRNA targets predicts functional non-conserved and non-canonical sites. Genome Biol 11: R90, 2010.

21. KelAE, Gössling E, Reuter I, CheremushkinE, Kel-Margoulis OV and Wingender E: MATCH: A tool for searching transcription factor binding sites in DNA sequences. Nucleic Acids Res 31: 3576-3579, 2003.

22. Prieto C, Risueño A, Fontanillo C and De Las RJ: Human gene coexpression landscape: Confident network derived from tissue transcriptomic profiles. PLoS One 3: e3911, 2008.

23. Quandt K, Frech K, Karas H, Wingender E and Werner T: MatInd and MatInspector: New fast and versatile tools for detection of consensus matches in nucleotide sequence data. Nucleic Acids Res 23: 4878-4884, 1995.

24. Jung EM, Choi KC and Jeung EB: Expression of calbindin-D28k is inversely correlated with proapototic gene expression in hydrogen peroxide-induced cell death in endometrial cancer cells. Int J Oncol 38: 1059-1066, 2011.

25. Sano M, Aoyagi K, Takahashi H, Kawamura T, Mabuchi T, Igaki H, Tachimori Y, Kato H, Ochiai A, Honda H, et al: Forkhead box A1 transcriptional pathway in KRT7-expressing esophageal squamous cell carcinomas with extensive lymph node metastasis. Int J Oncol 36: 321-330, 2010.

26. Chen YT, Panarelli NC, Piotti KC and Yantiss RK: Cancer-testis antigen expression in digestive tract carcinomas: Frequent expression in esophageal squamous cell carcinoma and its precursor lesions. Cancer Immunol Res 2: 480-486, 2014.

27. Madissoon E, Töhönen V, Vesterlund L, Katayama S, Unneberg P, Inzunza J, Hovatta $\mathrm{O}$ and Kere J: Differences in gene expression between mouse and human for dynamically regulated genes in early embryo. PLoS One 9: e102949, 2014.

28. Wong PP, Yeoh CC, Ahmad AS, Chelala C, Gillett C, Speirs V, Jones JL and Hurst HC: Identification of MAGEA antigens as causal players in the development of tamoxifen-resistant breast cancer. Oncogene 33: 4579-4588, 2014.

29. Liu CJ, Tsai MM, Tu HF, Lui MT, Cheng HW and Lin SC: miR-196a overexpression and miR-196a2 gene polymorphism are prognostic predictors of oral carcinomas. Ann Surg Oncol 20 (Suppl 3): S406-S414, 2013.

30. Lu YC, Chang JT, Huang YC, Huang CC, Chen WH, Lee LY, Huang BS, Chen YJ, Li HF and Cheng AJ: Combined determination of circulating miR-196a and miR-196b levels produces high sensitivity and specificity for early detection of oral cancer. Clin Biochem 48: 115-121, 2015. 
31. Saito K, Inagaki K, Kamimoto T, Ito Y, Sugita T, Nakajo S, Hirasawa A, Iwamaru A, Ishikura T, Hanaoka $\mathrm{H}$, et al: MicroRNA-196a is a putative diagnostic biomarker and therapeutic target for laryngeal cancer. PLoS One 8: e71480, 2013.

32. Severino P, Bruggemann H, Andreghetto FM, Camps C, Klingbeil Mde F, de Pereira WO, Soares RM, Moyses R, Wünsch-Filho V, Mathor MB, et al: MicroRNA expression profile in head and neck cancer: HOX-cluster embedded microRNA-196a and microRNA-10b dysregulation implicated in cell proliferation. BMC Cancer 13: 533, 2013.

33. Suh YE, Raulf N, Gäken J, Lawler K, Urbano TG, Bullenkamp J, Gobeil S, Huot J, Odell E and Tavassoli M: MicroRNA-196a promotes an oncogenic effect in head and neck cancer cells by suppressing annexin A1 and enhancing radioresistance. Int J Cancer 137: 1021-1034, 2015.

34. Ilmarinen T, Hagstrom J, Haglund C, Auvinen E, Leivo I, Pitkäranta A and Aaltonen LM: Low expression of nuclear Toll-like receptor 4 in laryngeal papillomas transforming into squamous cell carcinoma. Otolaryngol Head Neck Surg 151: 785-790, 2014

35. Makinen LK, Atula T, Häyry V, Jouhi L, Datta N, Lehtonen S, Ahmed A, Mäkitie AA, Haglund C and Hagström J: Predictive role of toll-like receptors 2,4 and 9 in oral tongue squamous cell carcinoma. Oral Oncol 51: 96-102, 2015.

36. Paluszczak J, Sarbak J, Kostrzewska-Poczekaj M, Kiwerska K Jarmuż-Szymczak M, Grenman R, Mielcarek-Kuchta D and Baer-Dubowska W: The negative regulators of Wnt pathway-DACH1, DKK1 and WIF1 are methylated in oral and oropharyngeal cancer and WIF1 methylation predicts shorter survival. Tumour Biol 36: 2855-2861, 2015.

37. Schussel JL, Kalinke LP, Sassi LM, de Oliveira BV, Pedruzzi PA, Olandoski M, Alvares LE, Garlet GP and Trevilatto PC: Expression and epigenetic regulation of DACT1 and DACT2 in oral squamous cell carcinoma. Cancer Biomark 15: 11-17, 2015.

38. Ting CM, Wong CK, Wong RN, Lo KW, Lee AW, Tsao GS, Lung ML and Mak NK: Role of STAT3/5 and Bcl-2/xL in 2 -methoxyestradiol-induced endoreduplication of nasopharyngeal carcinoma cells. Mol Carcinog 51: 963-972, 2012.
39. Iqbal MA, Gupta V, Gopinath P, Mazurek S and Bamezai RN: Pyruvate kinase M2 and cancer: An updated assessment. Febs Lett 588: 2685-2692, 2014.

40. Upadhyay M, Samal J, Kandpal M, Singh OV and Vivekanandan P: The Warburg effect: Insights from the past decade. Pharmacol Ther 137: 318-330, 2013.

41. Zhan C, Shi Y, Lu C and Wang Q: Pyruvate kinase M2 is highly correlated with the differentiation and the prognosis of esophageal squamous cell cancer. Dis Esophagus 26: 746-753, 2013.

42. Shi WY, Liu KD, Xu SG, Zhang JT, Yu LL, Xu KQ and Zhang TF: Gene expression analysis of lung cancer. Eur Rev Med Pharmacol Sci 18: 217-228, 2014.

43. Limame R, Op de Beek K, Lardon F, De Wever O and Pauwels P: Krüppel-like factors in cancer progression: Three fingers on the steering wheel. Oncotarget 5: 29-48, 2014.

44. Tie X, Han S, Meng L, Wang Y and Wu A: NFAT1 is highly expressed in, and regulates the invasion of, glioblastoma multiforme cells. PLoS One 8: e66008, 2013.

45. Braeuer RR, Zigler M, Kamiya T, Dobroff AS, Huang L, Choi W, McConkey DJ, Shoshan E, Mobley AK, Song R, et al: Galectin-3 contributes to melanoma growth and metastasis via regulation of NFAT1 and autotaxin. Cancer Res 72: 5757-5766, 2012

46. Nohata N, Sone Y, Hanazawa T, Fuse M, Kikkawa N, Yoshino H, Chiyomaru T, Kawakami K, Enokida H, Nakagawa M, et al: miR-1 as a tumor suppressive microRNA targeting TAGLN2 in head and neck squamous cell carcinoma. Oncotarget 2: 29-42, 2011.

47. Nohata N, Hanazawa T, Kikkawa N, Sakurai D, Sasaki K, Chiyomaru T, Kawakami K, Yoshino H, Enokida H, Nakagawa M, et al: Identification of novel molecular targets regulated by tumor suppressive miR-1/miR-133a in maxillary sinus squamous cell carcinoma. Int J Oncol 39: 1099-1107, 2011.

48. Wang F, Song G, Liu M, Li X and Tang H: miRNA-1 targets fibronectin1 and suppresses the migration and invasion of the HEp2 laryngeal squamous carcinoma cell line. FEBS Lett 585: 3263-3269, 2011

49. Wu CD, Kuo YS, Wu HC and Lin CT: MicroRNA-1 induces apoptosis by targeting prothymosin alpha in nasopharyngeal carcinoma cells. J Biomed Sci 18: 80, 2011. 